\title{
Eliminating the wave-function singularity for ultracold atoms by a similarity transformation
}

\author{
Péter Jeszenszki $\odot,{ }^{1,2}$ Ulrich Ebling $\odot,{ }^{1,2}$ Hongjun Luo $\odot,{ }^{3}$ Ali Alavi $\odot,{ }^{3,4}$ and Joachim Brand $\odot^{1,2,3, *}$ \\ ${ }^{1}$ Dodd-Walls Centre for Photonic and Quantum Technologies, PO Box 56, Dunedin 9056, New Zealand \\ ${ }^{2}$ New Zealand Institute for Advanced Study, and Centre for Theoretical Chemistry and Physics, Massey University, \\ Private Bag 102904 North Shore, Auckland 0745, New Zealand \\ ${ }^{3}$ Max Planck Institute for Solid State Research, Heisenbergstraße 1, 70569 Stuttgart, Germany \\ ${ }^{4}$ Department of Chemistry, University of Cambridge, Lensfield Road, Cambridge, CB2 1EW, United Kingdom
}

(Received 11 March 2020; revised 19 October 2020; accepted 23 October 2020; published 20 November 2020)

\begin{abstract}
A hyperbolic singularity in the wave function of $s$-wave interacting atoms is the root problem for any accurate numerical simulation. Here, we apply the transcorrelated method, whereby the wave-function singularity is explicitly described by a two-body Jastrow factor, and then folded into the Hamiltonian via a similarity transformation. The resulting nonsingular eigenfunctions are approximated by stochastic Fock-space diagonalization with energy errors scaling with $1 / M$ in the number $M$ of single-particle basis functions. The performance of the transcorrelated method is demonstrated on the example of strongly correlated fermions with unitary interactions. The current method provides the most accurate ground-state energies so far for three and four fermions in a rectangular box with periodic boundary conditions.
\end{abstract}

DOI: 10.1103/PhysRevResearch.2.043270

\section{INTRODUCTION}

Quantum gases make the study of strongly correlated many-body physics accessible [1], and can be probed with exquisite control in the many-particle [2-6] and few-particle $[7,8]$ regimes. At ultracold temperatures their interactions are accurately described by only the $s$-wave scattering length $a_{s}$ [9], or no parameters in the universal regime of unitary interactions [10]. Despite this apparent simplicity, it is nevertheless a great challenge to represent the complicated many-body wave functions in computational approaches [11]. Specifically, a $1 / r$ divergence when two particles with distance $r$ approach each other [12] introduces divergent short-range correlations into the wave function. While exact approaches are limited to four particles [13-17], computational approaches for larger particle numbers rely on lattice discretization with renormalized interactions [18] (employed at zero [19-22] and finite temperature [23-31]), the closely related renormalized contact interaction [32], finite-range pseudopotentials [33-35], or the more sophisticated effective Hamiltonian approaches [36,37]. By introducing an ultraviolet cutoff, these approaches do not accurately describe the short-range correlations, and suffer from slow convergence upon increasing the number of lattice sites or basis functions.

In this work we apply the transcorrelated method [38] to remove the short-range correlations from the wave function

\footnotetext{
*Corresponding author: j.brand@ massey.ac.nz
}

Published by the American Physical Society under the terms of the Creative Commons Attribution 4.0 International license. Further distribution of this work must maintain attribution to the author(s) and the published article's title, journal citation, and DOI. Open access publication funded by the Max Planck Society. by a similarity transformation of the many-body Hamiltonian. Previously, the transcorrelated method was applied to Coulomb-interacting electrons [39-42] and to ultracold atoms in one dimension [43]. In these cases the wave function is nonsingular but has a cusp, i.e., is continuous with a discontinuous first derivative [44]. Here, we extend the transcorrelated approach to the hyperbolic singularity and show that it is completely removed. The similarity-transformed, transcorrelated Hamiltonian is free from singular zero-range interactions, which are replaced by new two-body and three-body terms, and has nonsingular eigenfunctions. The advantages of the method are demonstrated by ground-state calculations with stochastic projective diagonalization in Fock space [45]. For a few fermions with unitary interactions we find that the error of the energy is the smallest among the available methodologies. Moreover, this error decays with $1 / M$, where $M$ is the number of the single-particle plane-wave basis functions. This is the fastest convergence rate so far.

This paper is organized as follows: Section II introduces the correlation factor for zero-range $s$-wave interactions and the transcorrelated transformation of the many-body Hamiltonian. Results on three and four fermions are described in Sec. III before concluding the main text with Sec. IV. Appendices provide many derivations and details starting with the real-space form of the correlation factor in Appendix A. Appendix B provides a derivation to show that the matrix elements of the similarity transformed FermiHuang pseudopotential between smooth functions vanish. The smoothness of the transcorrelated two-particle eigenfunctions is examined in Appendix C. Appendix D concerns the transcorrelated many-body Hamiltonian and provides it in second-quantized form, while Appendix E describes the algorithm used for evaluating an infinite sum that appears in the transcorrelated Hamiltonian. Appendix F finally provides details of the numerical calculations including parameters 
relating the the FCIQMC method (Appendix F 1), extrapolation procedures (Appendix F 2), and data (Appendix F 3), details about lattice renormalization procedures used for comparison (Appendix F 4), and about the exact and and approximate implementation of three-body interaction terms (Appendix F 5).

\section{CORRELATION FACTOR AND SIMILARITY TRANSFORMATION}

Zero-range $s$-wave interactions are characterized by the Bethe-Peierls boundary condition [12]

$$
\Psi\left(\mathbf{r}_{1}, \mathbf{r}_{2}, \ldots\right) \sim \frac{1}{r_{i j}}-\frac{1}{a_{s}}+\mathcal{O}\left(r_{i j}\right) \quad \text { for } \quad r_{i j} \rightarrow 0,
$$

where $r_{i j}=\left|\mathbf{r}_{i}-\mathbf{r}_{j}\right|$ is the distance between particles $i$ and $j$, and $\Psi$ is the many-body wave function. We aim to deal with the divergent short-range part with a Jastrow factor $e^{\tau}$ by writing

$$
\Psi\left(\mathbf{r}_{1}, \mathbf{r}_{2}, \ldots\right)=e^{\tau\left(\mathbf{r}_{1}, \mathbf{r}_{2}, \ldots\right)} \Phi\left(\mathbf{r}_{1}, \mathbf{r}_{2}, \ldots\right),
$$

which defines the transcorrelated wave function $\Phi . \tau$ is chosen as a sum of pair correlation factors $\tau\left(\mathbf{r}_{1}, \mathbf{r}_{2}, \ldots\right)=$ $\sum_{i<j} u\left(r_{i j}\right)$. Requiring

$$
u(r) \sim \text { const }-\ln \left(\frac{r}{a_{s}}\right)-\frac{r}{a_{s}}+\mathcal{O}\left(r^{2}\right)
$$

allows the Jastrow factor to carry the main part of the singular short-range correlation and leaves the transcorrelated wave function $\Phi$ nonsingular. Inserting the ansatz (2) into the Schrödinger equation $H \Psi=E \Psi$ and multiplying it with $e^{-\tau}$ from the left yields

$$
\tilde{H} \Phi=E \Phi,
$$

where $\tilde{H}=e^{-\tau} H e^{\tau}$ is the transcorrelated Hamiltonian [38].

It is convenient to define the correlation factor in momentum space, and we choose

$$
\tilde{u}(k)= \begin{cases}\frac{2 \pi^{2}}{k^{3}}+\frac{8 \pi}{a_{s} k^{4}} & \text { if } k \geqslant k_{c}, \\ 0 & \text { if } k<k_{c},\end{cases}
$$

where $k_{c}$ is a momentum cutoff. The real-space correlation factor is obtained by Fourier transform $u(r)=$ $(2 \pi)^{-2} \int_{0}^{\infty} d k \tilde{u}(k) k \sin (k r) / r$ and the corresponding Jastrow factor $\exp (u)$ is shown in Fig. 1. More details are provided in Appendix A, where it is shown that $u(r)$ satisfies Eq. (3). The momentum cutoff $k_{c}$ damps out the real-space $u(r)$ for large $r$. The idea is that long-range correlations in the transcorrelated wave function $\Phi$ can be effectively dealt with by the expansion in a Fock basis, as we will show, while the Jastrow factor $e^{\tau}$ very efficiently removes the singular short-range correlations.

For definiteness we consider a system of ultracold atoms of mass $m$ with Hamiltonian $H=H_{1}+V_{\mathrm{FH}}$, where $H_{1}=$ $\sum_{i}-\frac{\hbar^{2}}{2 m} \nabla_{i}^{2}+V_{\text {trap }}$ is the single-particle part with trapping potential $V_{\text {trap }}$. The zero-range $s$-wave interactions between atoms are represented by the Fermi-Huang pseudopotential

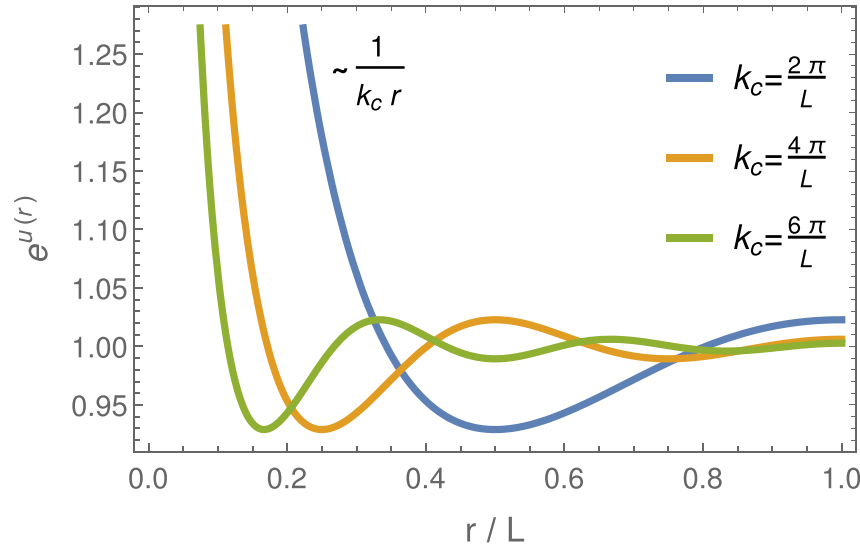

FIG. 1. The Jastrow factor $e^{u(r)}$ as a function of particle separation $r$ with unitary interaction at different values of the momentum cutoff. $L$ is the size of the computational box.

[46]

$$
V_{\mathrm{FH}}=g \sum_{i<j} \delta\left(\mathbf{r}_{i j}\right) \frac{\partial}{\partial r_{i j}} r_{i j},
$$

where $g=4 \pi \hbar^{2} a_{s} / m$ is the potential strength. The derivative term regularizes the otherwise pathological contact interaction and enforces the Bethe-Peierls boundary conditions of Eq. (1) $[46,47]$. This pseudopotential has been applied in exact $[13,48]$ and perturbative $[46]$ treatments, but it has a limitation in the Fock-state based approaches. As the Fock-state basis functions are smooth, the Fermi-Huang pseudopotential reduces to a simple Dirac-delta function, which is pathological in two and three dimensions [32,49-51]. It is suitable for use with the transcorrelated method, however, as long as the Jastrow factor $e^{\tau}$ is designed to fulfill Eq. (1).

The similarity transformation $\tilde{H}=e^{-\tau} H e^{\tau}$ is applied term by term and does not change simple functions of the coordinates because the correlation factor is local in the coordinates. The kinetic energy and $V_{\mathrm{FH}}$ contain coordinate derivatives and thus generate additional terms. Specifically, $e^{-\tau} V_{\mathrm{FH}} e^{\tau}=$ $V_{\mathrm{FH}}+\left[V_{\mathrm{FH}}, \tau\right]$ and, as we show in Appendix B,

$$
\left\langle\chi\left|\left[V_{\mathrm{FH}}, \tau\right]\right| \phi\right\rangle=\left\langle\chi\left|g \sum_{i<j} \delta\left(\mathbf{r}_{i j}\right) \frac{\partial u\left(r_{i j}\right)}{\partial r_{i j}} r_{i j}\right| \phi\right\rangle,
$$

for wave functions $\phi$ and $\chi$ that are bounded and have bounded first derivatives. In Appendix B it is shown that the matrix elements of the similarity-transformed Fermi-Huang pseudopotential $\left\langle\chi\left|e^{-\tau} V_{\mathrm{FH}} e^{\tau}\right| \phi\right\rangle$ vanish due to cancellation as long as the correlation factor $u(r)$ is chosen to have the appropriate short-range asymptotics of Eq. (3). Thus, the singular pseudopotential is removed and the transcorrelated Schrödinger equation (4) can be solved with a nonsingular wave function $\Phi$. This insight presents the main result of this paper.

The transcorrelated Hamiltonian still acquires terms that originate from the kinetic energy operator, and finally reads as

$$
\tilde{H}=H_{1}-\sum_{i}\left[\frac{1}{2} \nabla_{i}^{2} \tau+\left(\nabla_{i} \tau\right) \nabla_{i}+\frac{1}{2}\left(\nabla_{i} \tau\right)^{2}\right] \frac{\hbar^{2}}{m} .
$$


The new terms represent an effective interaction potential that is less singular than the Fermi-Huang pseudopotential. The leading singular term is $\left(\nabla_{i} \tau\right) \nabla_{i} \sim\left(\sum_{j} \mathbf{r}_{i j} / r_{i j}^{2}\right) \nabla_{i}$, which has a $1 / r$ divergence and is also non-Hermitian. Similar to the Coulomb potential it leads to a cusp feature, where the transcorrelated wave function $\Phi$ is continuous with a discontinuous first derivate (see in Appendix C). In momentum space, $\Phi$ thus decays with $1 / k^{4}$ for large $k$ instead of $1 / k^{2}$ for the original wave function $\Psi$. It is this rapid decay for large $k$ that makes it feasible to expand the problem in a plane-wave basis without the need for a renormalized (or running) coupling constant. The second-quantized form of the transcorrelated Hamiltonian in momentum space is presented in Appendix D.

Although the similarity transformation eliminates the singularity from the wave function without modifying the spectrum of the Hamiltonian, it introduces new challenges for numerical calculations. The non-Hermitian term $\left(\nabla_{i} \tau\right) \nabla_{i}$ prevents in general the variational minimization of the energy. However, the ground-state energy can still be found by projection techniques as proved in Ref. [41] and previously demonstrated in Refs. [41-43,52]. As a consequence of the non-Hermiticity, the approximate energies no longer provide an upper bound to the exact ground-state energy. Non-Hermitian terms are common in the transcorrelated and coupled-cluster methods and usually do not cause problems. Hypothetically, the projection onto a finite basis could lead to pairs of complex-conjugate eigenvalues with small imaginary parts in the vicinity of a accidental eigenvalue degeneracies. The stochastic projection used in this work would then fail to fully converge and resolve the near-degenerate eigenvalues. This is easy to diagnose but we have not encountered this situation so far. A removal strategy for complex eigenvalues was suggested in the context of coupled-cluster theory [53].

The terms $\frac{1}{2} \nabla_{i}^{2} \tau$ and $\frac{1}{2}\left(\nabla_{i} \tau\right)^{2}$ have leading $1 / r_{i j}^{2}$ and $1 / r_{i j} r_{i k}$ contributions, respectively, and partly compensate each other but leave an uncompensated three-body attraction. This long-range interaction represents a mediated three-body attraction that is familiar from Efimov physics [54,55]. It permits three-body bound states for resonantly interacting bosons but not for fermions. Three-body interactions are common in the transcorrelated method and were previously implemented for the Hubbard model [52] and electronic structure calculations in atoms [42]. Appendix F 5 presents details of the efficient numerical implementation of the three-body terms as well as a two-body approximation that saves up to a factor 4 in computer time while still maintaining high accuracy.

\section{NUMERICAL RESULTS}

For numerical calculations the transcorrelated Hamiltonian $\tilde{H}$ is expanded as a finite matrix in a Fock basis of antisymmetrized products of single-particle plane waves with a momentum cutoff. For two particles the ground-state energy is calculated with (numerically) exact diagonalization. For three and four fermions the full matrix diagonalization was not possible due to the enormous size of Hilbert space. Hence, we used a stochastic projection method known as full configuration interaction quantum Monte Carlo (FCIQMC) $[45,56]$ to

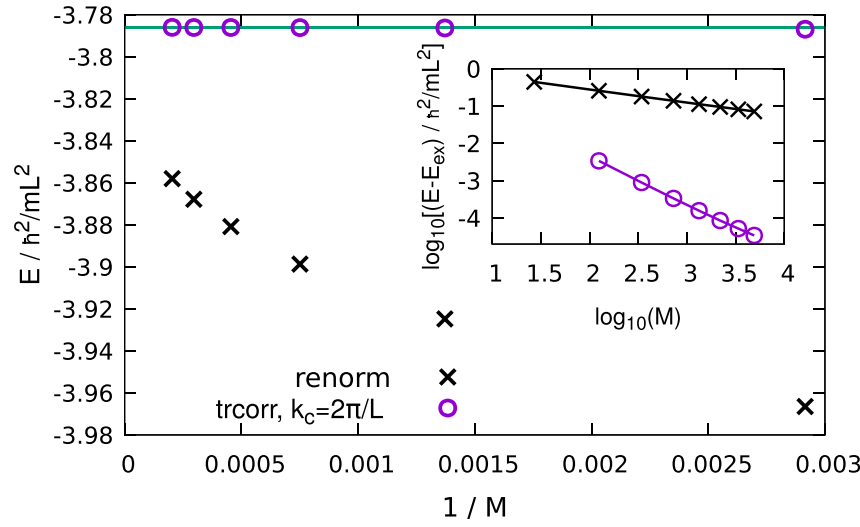

FIG. 2. Ground-state energy of two particles with unitary interactions $\left(1 / a_{s}=0\right)$ vs inverse size of single-particle basis $1 / M$ from the transcorrelated method (circles) and with renormalized Dirac delta (crosses). Horizontal green line: reference energy $E_{\mathrm{ex}}=$ $-3.786005 \hbar^{2} / m L^{2}$ [57]. Inset: Difference to the reference energy on a log-log plot indicating power-law scaling $\propto 1 / M^{1 / 3}$ for renormalization and $\propto 1 / M$ for the transcorrelated approach.

obtain the ground-state energies. A description of the method is provided in Appendix F.

Results for two particles are presented in Fig. 2. The energy is shown as a function of the inverse of the basisset size $M$, where $\sqrt[3]{M}$ is the number of single-particle plane-wave basis functions per linear dimension of the cube. Hence, the zero on the $x$ axis represents the complete basis-set limit. The transcorrelated energies are compared to standard lattice renormalization [18], where a running coupling constant $g_{0}$ is scaled with the number of lattice points $M$ as $g_{0}^{-1}=m / 4 \pi \hbar^{2} a_{s}-m K M^{1 / 3} / 4 \pi \hbar^{2} L(K=$ $2.442749607806335 \ldots$... [58]. It is not only seen that the transcorrelated method gives smaller errors by orders of magnitudes for the same $M$, but also that scaling of the errors with $M$ follows a faster power-law decay. For the renormalization approach, we find a scaling of $M^{-1 / 3}$ consistent with the previous results from lattice calculations [18,19,59]. In the transcorrelated approach, the error decays with $M^{-1}$. This is the same scaling as obtained for Coulomb-interacting systems, e.g., the homogeneous electron gas, which is consistent with the Coulomb-type nature of the transcorrelated Hamiltonian. The $M^{-1}$ scaling is the fastest scaling we found in the literature, and is shared, e.g., with the improved lattice action used for auxiliary field quantum Monte Carlo (AFQMC) calculations by Endres et al. [20] or the renormalized lattice Hamiltonian with "magic" dispersion relation discussed in Ref. [18].

Results for three fermions in the lowest-energy state with zero total momentum ${ }^{1}$ are shown in Fig. 3. While the $M^{-1}$ scaling can be observed for the "Endres AFQMC" values, the transcorrelated results are much more accurate already for very modest basis-set size and hardly distinguishable from the reference values on the scale of the figure. Moreover, in Appendix F 5 we show that approximate calculations avoiding

\footnotetext{
${ }^{1}$ The true ground state has finite momentum [33].
} 


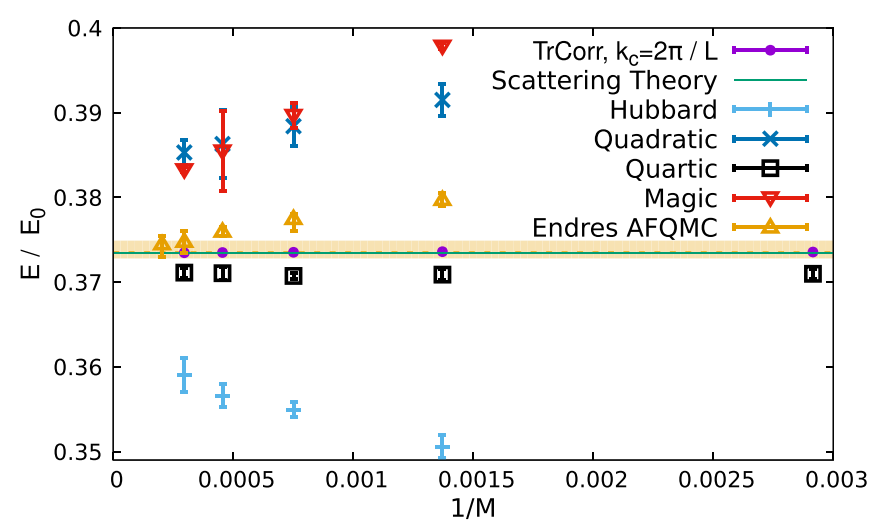

FIG. 3. Energy of zero-momentum ground state of two spin-up and one spin-down fermions with unitary interactions vs inverse size of single-particle basis $1 / M$. Transcorrelated ("TrCorr") are compared with semianalytical results from Ref. [60] ("scattering theory") and AFQMC ("Endres AFQMC") [20]. The horizontal yellow band marks the standard error of the extrapolated AFQMC results. Renormalized lattice calculations with FCIQMC using different single-particle dispersions: "Hubbard," "quadratic," and "quartic" as in Ref. [19] and the "magic" dispersion from Eqs. (122) and (124) in Ref. [18]. $E_{0}=4 \pi^{2} \hbar^{2} / m L^{2}$ is the noninteracting energy.

the numerically expensive three-body excitations achieve the same accuracy within our statistical errors.

Figure 3 also shows renormalized lattice calculations with different single-particle dispersion relations as discussed in Refs. [18,19] obtained with FCIQMC. Since they are expected to show slower scaling than $M^{-1}$, the energy dependence does not appear linear in Fig. 3. The renormalized lattice method scales with $M^{-1 / 3}$ when using a Hubbard, quadratic, or quartic dispersion, and $M^{-2 / 3}$ for a "magic" dispersion [18].

The transcorrelated energies for three particles are shown again in Fig. 4 with a magnified energy scale and with differ-

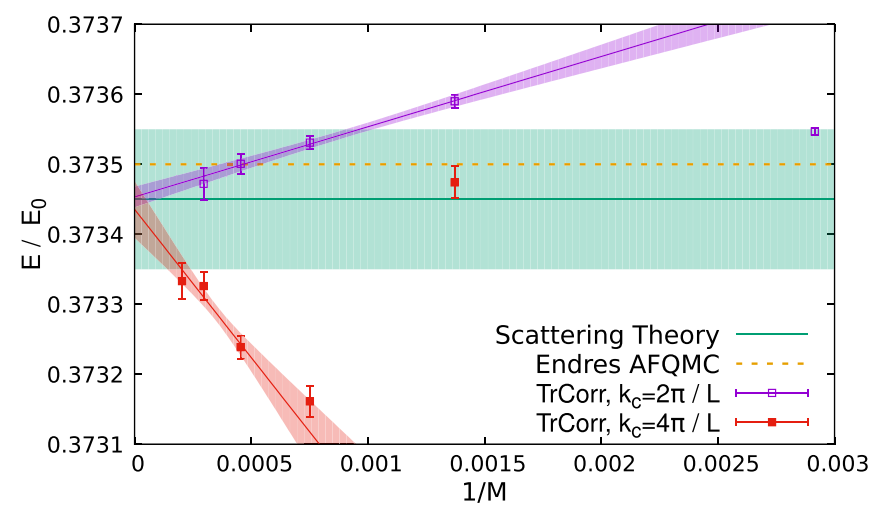

FIG. 4. Detail from Fig. 3 at enlarged scale. The error bars of the transcorrelated results show the stochastic errors from the FCIQMC method. The reference value from Ref. [60] ("scattering theory") is marked with a horizontal line and error band in green, extrapolated result from Endres AFQMC [20] as a dashed yellow line (error not shown). The diagonal red and purple lines and bands indicate the linear fits and $1 \sigma$ confidence bands obtained from $\chi^{2}$ fitting of the transcorrelated FCIQMC (TrCorr) results (four largest $M$ values), respectively. For details, see Appendix F 2.

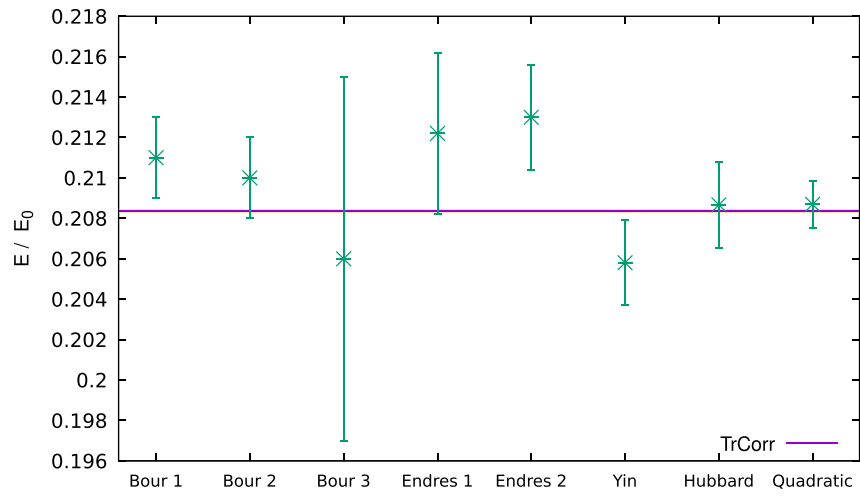

FIG. 5. Ground-state energy of four fermions extrapolated to infinite basis-set limit. The horizontal (purple) line (TrCorr) shows the transcorrelated result $E / E_{0}=0.208339 \pm 0.000094$ with the error indicated by yellow band. Results from Ref. [59] with Hamiltonian lattice 1 ("Bour 1") and 2 ("Bour 2") and AFQMC with Euclidian lattice ("Bour 3") are shown alongside AFQMC results from Ref. [20] with $\mathcal{O}(4)$ ("Endres 1") and $\mathcal{O}(5)$ ("Endres 2") scaling, explicitly correlated Gaussian ("Yin") [33], and renormalized lattice calculations following Ref. [19] using "Hubbard" and "quadratic" dispersion relations. For the numerical values, see Table III in Appendix F.

ent momentum cutoffs $k_{c}$ in the correlation factor of Eq. (5). It is seen that the asymptotic regime of $M^{-1}$ scaling of the energy error is only reached for the larger basis-set sizes. With the known asymptotic scaling properties we can determine the energies in the infinite basis-set limit by extrapolation. The extrapolations with two different $k_{c}$ values are seen to be consistent with each other as well as with the literature results from scattering theory [60] and AFQMC [20], while they have much smaller error bars than previous results. As the final value for the lowest energy with zero total momentum for three fermions we obtain $E / E_{0}=0.373453 \pm 0.000034$ using $k_{c}=2 \pi / L$, where $E_{0}$ is the three-particle energy without interaction between the particles. Compared to the results of Endres et al. [20] of $E / E_{0}=0.3735(+0.0014 /-0.0007)$ the error is reduced by more than an order of magnitude.

The results from transcorrelated and renormalized calculations for a four-fermion system are shown in Fig. 5, where they are compared to literature results with lattice discretization (exact diagonalization and AFQMC) and explicitly correlated basis-set approaches. Despite the several orders-ofmagnitude larger Hilbert space $\left(\sim 10^{14}\right)$ we obtain bias-free results from FCIQMC by using the initiator approximation and bias removal by increasing the walker number [61]. The combined stochastic and extrapolation error of the transcorrelated approach is about two orders of magnitude smaller than the best existing literature values (for details and numerical values see Appendix F). This result showcases the significance of an explicit treatment of the wave-function singularity for improving the accuracy of numerical calculations.

\section{CONCLUSIONS}

The approach presented here can be easily extended to include trapping potentials or external gauge fields. The transcorrelated method is thus well suited for highly precise 
calculations on correlated few-atom systems in microtraps $[7,8]$. Extensions to larger particle numbers are feasible and have already been demonstrated with FCIQMC in weakly correlated regimes [62], while recent developments of FCIQMC like the adaptive shift method [63] can help in strongly correlated regimes. The approach can be applied to lowdimensional systems, and while it has already proven useful in one dimension [43], the two-dimensional case is an objective for the future. The transcorrelated method could also be employed with extensions of FCIQMC for finite-temperature calculations with density-matrix Monte Carlo [64], or realtime evolution of closed [65] or open quantum systems [66]. Beyond the specific numerical approach, we expect that the transcorrelated formalism brings new insight into the treatment of the singularity in the wave function and that it provides a useful theoretical tool in other perturbative and exact computational approaches.

\section{ACKNOWLEDGMENTS}

The authors thank E. Pahl, A. Punnett, and P. López Riós for discussions, L. Pricoupenko and Y. Castin for raw data relating to Ref. [60], D. Blume for a careful reading of the manuscript and for pointing out Ref. [33], and K. Guther for the help with the integration of code into the NECI program package. P.J. thanks the Max Planck Institute for Solid State Research for hospitality during a research stay where this project was started. This work was supported by the Marsden Fund of New Zealand (Contract No. MAU1604), from government funding managed by the Royal Society Te Apārangi. We also acknowledge support by the NeSI high-performance computing facilities.

\section{APPENDIX A: CORRELATION FACTOR IN REAL SPACE}

In this Appendix, we examine the real-space form of the correlation factor,

$$
\tilde{u}(k)= \begin{cases}\frac{2 \pi^{2}}{k^{3}}+\frac{8 \pi}{a_{s} k^{4}} & \text { if } k \geqslant k_{c}, \\ 0 & \text { if } k<k_{c} .\end{cases}
$$

The Fourier transform of function (A1) can be calculated analytically:

$$
\begin{aligned}
& u(r)=\int_{-\infty}^{\infty} d k^{3} \tilde{u}(|\mathbf{k}|) \frac{e^{i \mathbf{k} \cdot \mathbf{r}}}{(2 \pi)^{3}}=v(r)+\frac{8 \pi}{a_{s}} w(r), \\
& v(r)=\frac{\sin \left(k_{c} r\right)}{k_{c} r}-\operatorname{Ci}\left(k_{c} r\right), \\
& w(r)=\frac{\cos \left(k_{c} r\right)}{4 k_{c} \pi^{2}}+\frac{\sin \left(k_{c} r\right)}{4 k_{c}^{2} \pi^{2} r}+\frac{r}{4 \pi^{2}} \operatorname{si}\left(k_{c} r\right),
\end{aligned}
$$

where $\operatorname{Ci}(x)=-\int_{x}^{\infty} \frac{\cos (t) d t}{t}$ is the cosine integral and $\operatorname{si}(x)=$ $-\int_{x}^{\infty} \frac{\sin (t) d t}{t}$ is the sine integral. The boundary condition can be reproduced by expanding function $v(r)$ and $w(r)$ in Taylor series around $r=0$ :

$$
\begin{aligned}
& v(r)=-\ln \left(k_{c} r\right)+1-\gamma+\mathcal{O}\left(k_{c}^{2} r^{2}\right), \\
& w(r)=\frac{1}{2 \pi^{2} k_{c}}-\frac{r}{8 \pi}+\mathcal{O}\left(k_{c}^{2} r^{2}\right), \\
& u(r)=-\ln \left(k_{c} r\right)+1-\gamma+\frac{4}{\pi a_{s} k_{c}}-\frac{r}{a_{s}}+\mathcal{O}\left(k_{c}^{2} r^{2}\right),
\end{aligned}
$$

where $\gamma$ is the Euler-Mascheroni constant. Calculating $e^{u(r)}$, we obtain back the hyperbolic singularity for the Jastrow factor,

$$
e^{u(r)}=e^{1-\gamma+\frac{4}{a_{s} k_{c} \pi}}\left[\frac{1}{k_{c} r}-\frac{1}{k_{c} a_{s}}+\mathcal{O}\left(k_{c} r\right)\right] .
$$

\section{APPENDIX B: MATRIX ELEMENTS OF THE TRANSCORRELATED FERMI-HUANG PSEUDOPOTENTIAL}

We consider the matrix element of the transcorrelated Fermi-Huang pseudopotential and show that it vanishes, if evaluated with wave functions that are bounded and have a bounded first derivative almost everywhere.

In order to show that, let us consider the transcorrelated Fermi-Huang pseudopotential

$$
e^{-\tau} V_{\mathrm{FH}} e^{\tau}=V_{\mathrm{FH}}+\left[V_{\mathrm{FH}}, \tau\right],
$$

where the commutator can be evaluated if we apply the substitution $V_{\mathrm{FH}}=g \sum_{i<j} \delta\left(\mathbf{r}_{i j}\right) \frac{\partial}{\partial r_{i j}} r_{i j}$,

$$
\left[V_{\mathrm{FH}}, \tau\right]=g \sum_{i<j} \delta\left(\mathbf{r}_{i j}\right) \frac{\partial \tau}{\partial r_{i j}} r_{i j}
$$

The partial derivative with respect to the separation $r_{i j}=$ $\left|\mathbf{r}_{i}-\mathbf{r}_{j}\right|$ is defined in the usual way, where both particles $i$ and $j$ move while the center of mass $\frac{1}{2}\left(\mathbf{r}_{i}+\mathbf{r}_{j}\right)$ is held constant, as are the orientation of the vector $\mathbf{r}_{i j}=\mathbf{r}_{i}-\mathbf{r}_{j}$, and all other particle coordinate vectors $\mathbf{r}_{k}$ for $k \neq i, k \neq j$. Since $\tau$ depends on the separations of all particle pairs, the chain rule will generate many terms, most of which, however, vanish.

In order to evaluate the derivative $\frac{\partial \tau}{\partial r_{i j}}$, let us substitute in the expansion of $\tau$ in pair correlation functions

$$
\tau=\sum_{i<j} u\left(r_{i j}\right)
$$

into $\frac{\partial \tau}{\partial r_{i j}}$,

$$
\begin{aligned}
\frac{\partial \tau}{\partial r_{i j}}= & \frac{\partial u\left(r_{i j}\right)}{\partial r_{i j}}+\sum_{l}^{i<l \neq j} \frac{\partial u\left(r_{i l}\right)}{\partial r_{i j}}+\sum_{k}^{i \neq k<j} \frac{\partial u\left(r_{k j}\right)}{\partial r_{i j}} \\
& +\sum_{k<l}^{i \neq k, j \neq l} \frac{\partial u\left(r_{k l}\right)}{\partial r_{i j}} .
\end{aligned}
$$


The last term on the right-hand side is zero as $r_{k l}$ does not depend on $r_{i j}$. For the second and the third terms on the righthand side we can apply the chain rule

$$
\begin{gathered}
\frac{\partial u\left(r_{i l}\right)}{\partial r_{i j}}=\frac{\partial u\left(r_{i l}\right)}{\partial r_{i l}} \underbrace{\sum_{p=1}^{3} \frac{\partial r_{i l}}{\partial r_{i, p}} \frac{\partial r_{i, p}}{\partial r_{i j}}}_{\cos \theta_{j i l} / 2}, \\
\frac{\partial u\left(r_{k j}\right)}{\partial r_{i j}}=\frac{\partial u\left(r_{k j}\right)}{\partial r_{k j}} \underbrace{\sum_{p=1}^{3} \frac{\partial r_{k j}}{\partial r_{j, p}} \frac{\partial r_{j, p}}{\partial r_{i j}}}_{\cos \theta_{i j k} / 2},
\end{gathered}
$$

where index $p$ goes through the three spatial directions and $\theta_{j i l}$ is the angle between $\mathbf{r}_{j i}$ and $\mathbf{r}_{i l}$.

Using the short-range behavior of the correlation factor

$$
u(r)=1-\ln \left(\frac{r}{a_{s}}\right)-\frac{r}{a_{s}}+\mathcal{O}\left(r^{2}\right),
$$

the first derivative of $u(r)$ can be evaluated for short interparticle separations

$$
\frac{d u(r)}{d r}=-\frac{1}{r}-\frac{1}{a_{s}}+\mathcal{O}(r) .
$$

Substituting Eqs. (B5)-(B8) into Eq. (B4), the explicit expression can be obtained for $\partial \tau / \partial r_{i j}$ :

$$
\begin{aligned}
\frac{\partial \tau}{\partial r_{i j}}= & -\frac{1}{r_{i j}}-\sum_{l}^{i<l \neq j} \frac{\cos \theta_{j i l}}{2 r_{i l}}-\sum_{k}^{i \neq k<j} \frac{\cos \theta_{i j k}}{2 r_{k j}} \\
& -\frac{1}{a_{s}}\left(1+\sum_{l}^{i<l \neq j} \frac{\cos \theta_{j i l}}{2}+\sum_{k}^{i \neq k<j} \frac{\cos \theta_{i j k}}{2}\right)+\mathcal{O}\left(r_{i j}\right) .
\end{aligned}
$$

Using the expression above, we can evaluate the matrix element of the commutator expression (B2), where the delta function restricts the spatial integration to short interparticle separations

$$
\begin{aligned}
\left\langle\chi\left|g \sum_{i<j} \delta\left(\mathbf{r}_{i j}\right) \frac{\partial \tau}{\partial r_{i j}} r_{i j}\right| \phi\right\rangle \\
=g \sum_{i<j}\left[-\left\langle\chi\left|\delta\left(\mathbf{r}_{i j}\right)\right| \phi\right\rangle-\sum_{l}^{i<l \neq j}\left\langle\chi\left|\frac{\delta\left(\mathbf{r}_{i j}\right) r_{i j} \cos \theta_{j i l}}{2 r_{i l}}\right| \phi\right\rangle\right. \\
\quad-\sum_{l}^{i \neq k<j}\left\langle\chi\left|\frac{\delta\left(\mathbf{r}_{i j}\right) r_{i j} \cos \theta_{i j k}}{2 r_{k j}}\right| \phi\right\rangle+\left\langle\chi\left|\delta\left(\mathbf{r}_{i j}\right) r_{i j} \mathcal{O}\left(r_{i j}\right)\right| \phi\right\rangle \\
\left.-\left(\frac{1}{a_{s}}+\sum_{l} \frac{\cos \theta_{j i l}}{2 a_{s}}+\sum_{k}^{i \neq k<j} \frac{\cos \theta_{i j k}}{2 a_{s}}\right)\left\langle\chi\left|\delta\left(\mathbf{r}_{i j}\right) r_{i j}\right| \phi\right\rangle\right] .
\end{aligned}
$$

Assuming that the functions $\chi$ and $\phi$ are bounded, the last two terms in Eq. (B10) are zero as $\delta\left(\mathbf{r}_{i j}\right) r_{i j}$ gives zero after performing the integral either for $\mathbf{r}_{i}$ or $\mathbf{r}_{j}$. Although the integrands in the second and the third term on the right-hand side of Eq. (B10) can be finite at the coalescence points $\mathbf{r}_{i}=\mathbf{r}_{l}$ and $\mathbf{r}_{k}=\mathbf{r}_{j}$, they are still zero everywhere else. Since the coalescence points form a set of measure zero, these terms yield zero after integrating over the remaining variables. This leads to a matrix element of the Dirac delta function:

$$
\left\langle\chi\left|g \sum_{i<j} \delta\left(\mathbf{r}_{i j}\right) \frac{\partial \tau}{\partial r_{i j}} r_{i j}\right| \phi\right\rangle=-g \sum_{i<j}\left\langle\chi\left|\delta\left(\mathbf{r}_{i j}\right)\right| \phi\right\rangle .
$$

Due to the bounded nature of the functions $\phi$ and $\chi$, the matrix element of the Fermi-Huang pseudopotential also reduces to the matrix element of the Dirac-delta function, but with the opposite sign,

$$
\begin{aligned}
& \left\langle\chi\left|g \sum_{i<j} \delta\left(\mathbf{r}_{i j}\right) \frac{\partial}{\partial r_{i j}} r_{i j}\right| \phi\right\rangle \\
& \quad=g \sum_{i<j}\left\langle\chi\left|\delta\left(\mathbf{r}_{i j}\right)\right| \phi\right\rangle+g \sum_{i<j} \underbrace{\left\langle\chi\left|\delta\left(\mathbf{r}_{i j}\right) r_{i j} \frac{\partial}{\partial r_{i j}}\right| \phi\right\rangle}_{0},
\end{aligned}
$$

where we have assumed that $\chi$ and $\partial \phi / \partial r_{i j}$ are bounded. Equation (B12) shows that a matrix representation of the the (physically meaningful) Fermi-Huang pseudopotential with sufficiently smooth (and bounded) basis functions is equivalent to the bare Dirac-delta pseudopotential, which is pathological in the sense that the infinite basis-set limit does not exist. After the transcorrelated similarity transformation, however, we obtain the two matrix elements (B11) and (B12), which cancel each other and thus eliminate the irregular behavior in the matrix representation. Combining Eqs. (B1), (B2), (B11), and (B12) we finally obtain

$$
\left\langle\chi\left|e^{-\tau} V_{\mathrm{FH}} e^{\tau}\right| \phi\right\rangle=\left\langle\chi\left|V_{\mathrm{FH}}+\left[V_{\mathrm{FH}}, \tau\right]\right| \phi\right\rangle=0 .
$$

\section{APPENDIX C: SMOOTHNESS OF THE TRANSCORRELATED EIGENFUNCTION FOR TWO PARTICLES}

In this Appendix we investigate the transcorrelated eigenfunction for two bosons or distinguishable particles with the same mass (e.g., fermions with different spin quantum number). We show that the singularity is reduced in the transcorrelated Hamiltonian due to the similarity transformation. Consequently, the transcorrelated eigenfunctions are not singular, there is only a cusp at the particle-particle coalescence point.

We consider the two-particle Hamiltonian without trapping potential $\left(V_{\text {trap }}=0\right)$

$$
H=-\frac{\hbar^{2}}{2 m} \nabla_{\uparrow}^{2}-\frac{\hbar^{2}}{2 m} \nabla_{\downarrow}^{2}+g \delta\left(\mathbf{r}_{\uparrow}-\mathbf{r}_{\downarrow}\right) \frac{\partial}{\partial\left|\mathbf{r}_{\uparrow}-\mathbf{r}_{\downarrow}\right|}\left|\mathbf{r}_{\uparrow}-\mathbf{r}_{\downarrow}\right|,
$$

where $\uparrow$ and $\downarrow$ label the two particles. Separating the center of mass from the relative motion coordinates, we obtain

$$
H_{\mathrm{rel}}=-\frac{\hbar^{2}}{2 \mu} \nabla^{2}+g \delta(\mathbf{r}) \frac{\partial}{\partial r} r
$$

where $\mathbf{r}=\mathbf{r}_{\uparrow}-\mathbf{r}_{\downarrow}, \mu=m / 2$, and the center of mass is described by free-particle motion. 
Applying the transcorrelated similarity transformation to the relative-motion Hamiltoninan of Eq. (C1) yields

$$
\begin{aligned}
\tilde{H}_{\mathrm{rel}}= & e^{-\tau} H_{\mathrm{rel}} e^{\tau} \\
\tilde{H}_{\mathrm{rel}}= & -\frac{\hbar^{2}}{2 \mu} \nabla^{2}-\frac{\hbar^{2}}{\mu}\left[\frac{1}{2} \nabla^{2} \tau+(\nabla \tau) \nabla+\frac{1}{2}(\nabla \tau)^{2}\right] \\
& +g \delta(\mathbf{r})\left[\frac{\partial}{\partial r} r+r\left(\frac{\partial \tau}{\partial r}\right)\right] .
\end{aligned}
$$

Using Eqs. (B3) and (B7), $\tau$ can be given explicitly at small interparticle separation

$$
\tau=1-\ln \left(\frac{r}{a_{s}}\right)-\frac{r}{a_{s}}+\mathcal{O}\left(r^{2}\right),
$$

with which the derivatives of $\tau$ in Eq. (C2) can be expressed as

$$
\begin{aligned}
\frac{\partial \tau}{\partial r} & =-\frac{1}{r}-\frac{1}{a_{s}}+\mathcal{O}(r), \\
\nabla \tau & =-\frac{\mathbf{r}}{r^{2}}-\frac{\mathbf{r}}{a_{s} r}+\mathcal{O}(\mathbf{r}), \\
\nabla^{2} \tau & =-\frac{1}{r^{2}}-\frac{2}{a_{s} r}+\mathcal{O}\left(r^{0}\right) .
\end{aligned}
$$

Substituting back into Eq. (C2), an explicit expression for the Hamiltonian can be obtained for short distances

$$
\begin{aligned}
\tilde{H}_{\mathrm{rel}} & =-\frac{\hbar^{2}}{2 \mu} \nabla^{2}+\frac{\hbar^{2}}{\mu}\left(\frac{1}{r}+\frac{1}{a_{s}}\right) \frac{\mathbf{r}}{r} \nabla \\
& +g \delta(\mathbf{r})\left(r \frac{\partial}{\partial r}-\frac{r}{a_{s}}+\mathcal{O}\left(r^{2}\right)\right)+\mathcal{O}\left(r^{0}\right) .
\end{aligned}
$$

In order to obtain the transcorrelated eigenfunction, let us substitute the Hamiltonian into the Schrödinger equation

$$
\begin{aligned}
-\frac{\hbar^{2}}{2 \mu} \nabla^{2} \phi(\mathbf{r}) & +\frac{\hbar^{2}}{\mu}\left(\frac{1}{r}+\frac{1}{a_{s}}\right) \frac{\mathbf{r}}{r} \nabla \phi(\mathbf{r}) \\
& +g \delta(\mathbf{r})\left(r \frac{\partial}{\partial r}-\frac{r}{a_{s}}+\mathcal{O}\left(r^{2}\right)\right) \phi(\mathbf{r})=E^{\prime} \phi(\mathbf{r}),
\end{aligned}
$$

where $E^{\prime}=E+\mathcal{O}\left(r^{0}\right)$. Due to the spherical symmetry we can transform the differential equation into polar coordinates and consider only $s$-wave solutions

$$
\begin{aligned}
& -\frac{\partial^{2} \phi(r)}{\partial r^{2}}+\frac{2}{a_{s}} \frac{\partial \phi(r)}{\partial r}+\frac{g \mu}{2 \pi \hbar^{2} r} \delta(r)\left[\frac{\partial}{\partial r}+\frac{1}{a_{s}}+\mathcal{O}\left(r^{2}\right)\right] \phi(r) \\
& =\frac{2 \mu E^{\prime}}{\hbar^{2}} \phi(r) .
\end{aligned}
$$

The differential equation can be solved for small interparticle separation

$$
\phi(r) \stackrel{r \rightarrow 0}{=} e^{\frac{r}{a_{s}}}\left(c_{1} e^{\sqrt[r]{\frac{\hbar^{2}}{2 \mu a_{s}^{2}}-E^{\prime}}}+c_{2} e^{-r \sqrt{\frac{\hbar^{2}}{2 \mu a_{s}^{2}}-E^{\prime}}}\right),
$$

where $c_{1}, c_{2}$, and $E^{\prime}$ can be determined only if we know the solution in the whole space.

Differentiating the wave function we notice that it has a linear term

$$
\phi(r)=c_{1}+c_{2}+b r+\mathcal{O}\left(r^{2}\right),
$$

where the prefactor $b$, before the linear term, is

$$
b=\phi^{\prime}(0)=\frac{c_{1}+c_{2}}{a_{s}}+\left(c_{1}-c_{2}\right) \sqrt{\frac{\hbar^{2}}{2 \mu a_{s}^{2}}-E^{\prime}} .
$$

Considering the spherical symmetry, we obtain a function which goes linearly to $c_{1}+c_{2}$ around the origin and forms a cusp. This function is not singular and continuous, however, its first derivative is discontinuous. Therefore, the transcorrelated transformation smooths the wave function from a hyperbolic singularity $(\sim 1 / r)$ to a cusp feature.

\section{APPENDIX D: SECOND-QUANTIZED FORM OF THE TRANSCORRELATED HAMILTONIAN}

In this Appendix, we give an explicit expression for the second-quantized form of the transcorrelated Hamiltonian in a rectangular box with periodic boundary conditions.

Starting from the full Hamiltonian of Eq. (7) of the main text we write

$$
H=H_{\mathrm{k}}+\sum_{i} V_{\text {trap }}\left(\mathbf{r}_{i}\right)+V_{\mathrm{FH}},
$$

where

$$
H_{\mathrm{k}}=\sum_{i}-\frac{\hbar^{2}}{2 m} \nabla_{i}^{2}
$$

is the kinetic energy operator. Under the transcorrelated similarity transformation

$$
\begin{aligned}
\tilde{H} & =e^{-\tau} H e^{\tau}=e^{-\tau} H_{\mathrm{k}} e^{\tau}+\sum_{i} V_{\text {trap }}\left(\mathbf{r}_{i}\right) \\
& =\tilde{H}_{\mathrm{k}}+\sum_{i} V_{\text {trap }}\left(\mathbf{r}_{i}\right),
\end{aligned}
$$

where we assume that will only apply the transcorrelated Hamiltonian in the domain of bounded and almosteverywhere differentiable functions, under which conditions the Fermi-Huang pseudopotential disappears according to Appendix B. The trapping potential is unchanged by the similarity transformation because it is a diagonal operator in coordinate space.

The transcorrelated kinetic energy operator obtains additional terms, as already discussed [see Eq. (8) in the main text]:

$$
\tilde{H}_{\mathrm{k}}=-\frac{\hbar^{2}}{2 m} \sum_{i}\left[\nabla_{i}^{2}+\frac{1}{2} \nabla_{i}^{2} \tau+\left(\nabla_{i} \tau\right) \nabla_{i}+\frac{1}{2}\left(\nabla_{i} \tau\right)^{2}\right] .
$$

Assuming a box with side length $L$ and periodic boundary conditions, we can introduce the usual plane-wave 
single-particle basis functions. Following the description in Ref. [41], the second-quantized form of the transcorrelated kinetic energy operator is easily determined as

$$
\begin{aligned}
\tilde{H}_{\mathbf{k}}= & \frac{\hbar^{2}}{2 m} \sum_{\mathbf{k} \sigma} k^{2} a_{\mathbf{k}, \sigma}^{\dagger} a_{\mathbf{k}, \sigma} \\
& +\sum_{\substack{\mathbf{p q k} \\
\sigma \sigma^{\prime}}} T_{\mathbf{p q k}} \Theta_{\sigma \sigma^{\prime}} a_{\mathbf{p}-\mathbf{k}, \sigma}^{\dagger} a_{\mathbf{q}+\mathbf{k}, \sigma^{\prime}}^{\dagger} a_{\mathbf{q}, \sigma^{\prime}} a_{\mathbf{p}, \sigma} \\
& +\sum_{\substack{\mathbf{p q s} \\
\mathbf{k} \mathbf{k}^{\prime}}} Q_{\mathbf{k} \mathbf{k}^{\prime}} \Theta_{\sigma \sigma^{\prime}} a_{\mathbf{p}-\mathbf{k}, \sigma}^{\dagger} a_{\mathbf{q}+\mathbf{k}^{\prime}, \sigma}^{\dagger} a_{\mathbf{s}+\mathbf{k}-\mathbf{k}^{\prime}, \sigma^{\prime}}^{\dagger} a_{\mathbf{s}, \sigma^{\prime}} a_{\mathbf{q}, \sigma} a_{\mathbf{p}, \sigma}
\end{aligned}
$$

where $a_{\mathbf{k}, \sigma}^{\dagger}$ creates a one-particle plane-wave state with momentum $\mathbf{k}$ and spin $\sigma$ and $\Theta_{\sigma \sigma^{\prime}}=\delta_{\sigma \sigma^{\prime}}$ for bosons and $\Theta_{\sigma \sigma^{\prime}}=$ $1-\delta_{\sigma \sigma^{\prime}}$ for fermions. The tensors $\mathbf{T}$ and $\mathbf{Q}$ can be expressed explicitly as

$$
\begin{aligned}
T_{\mathbf{p q k}} & =\frac{\hbar^{2}}{m L^{3}}\left(k^{2} \tilde{u}(k)-(\mathbf{p}-\mathbf{q}) \mathbf{k} \tilde{u}(k)+\frac{W(\mathbf{k})}{L^{3}}\right), \\
W(\mathbf{k}) & =\sum_{\mathbf{k}^{\prime}}\left(\mathbf{k}-\mathbf{k}^{\prime}\right) \mathbf{k}^{\prime} \tilde{u}\left(\left|\mathbf{k}-\mathbf{k}^{\prime}\right|\right) \tilde{u}\left(k^{\prime}\right), \\
Q_{\mathbf{k} \mathbf{k}^{\prime}} & =-\frac{\mathbf{k}^{\prime} \mathbf{k} \tilde{u}(k) \tilde{u}\left(k^{\prime}\right) \hbar^{2}}{2 m L^{6}} .
\end{aligned}
$$

\section{APPENDIX E: NUMERICAL EVALUATION OF THE INFINITE SUMMATION IN EQ. (D5)}

In this Appendix we describe the algorithm that we have used for evaluating the infinite sum in Eq. (D5). First, let us realize that we can restrict the indices in the summation from below due to momentum cutoff in the correlation factor (A1):

$$
W(\mathbf{k})=\sum_{\mathbf{k}^{\prime}}^{k^{\prime},\left|\mathbf{k}-\mathbf{k}^{\prime}\right| \geqslant k_{c}}\left(\mathbf{k}-\mathbf{k}^{\prime}\right) \mathbf{k}^{\prime} \tilde{u}\left(\left|\mathbf{k}-\mathbf{k}^{\prime}\right|\right) \tilde{u}\left(k^{\prime}\right) .
$$

As we can see in Eq. (E1), the summation goes to infinity, which prohibits the exact evaluation. However, an accurate approximate value can be obtained if we partition the summation in Eq. (E1) to a summation inside a sphere with radius $k_{\text {int }}$ and a summation outside this sphere:

$$
\begin{aligned}
W(\mathbf{k}) & =P\left(\mathbf{k}, k_{\text {int }}\right)+R\left(\mathbf{k}, k_{\text {int }}\right), \\
P\left(\mathbf{k}, k_{\text {int }}\right) & =\sum_{\mathbf{k}^{\prime}}^{k_{\text {int }}>k^{\prime},\left|\mathbf{k}-\mathbf{k}^{\prime}\right| \geqslant k_{c}} w\left(\mathbf{k}, \mathbf{k}^{\prime}\right), \\
R\left(\mathbf{k}, k_{\text {int }}\right) & =\sum_{\mathbf{k}^{\prime}}^{k^{\prime},\left|\mathbf{k}-\mathbf{k}^{\prime}\right| \geqslant k_{\text {int }}} w\left(\mathbf{k}, \mathbf{k}^{\prime}\right), \\
w\left(\mathbf{k}, \mathbf{k}^{\prime}\right) & =\left(\mathbf{k}-\mathbf{k}^{\prime}\right) \mathbf{k}^{\prime} \tilde{u}\left(\left|\mathbf{k}-\mathbf{k}^{\prime}\right|\right) \tilde{u}\left(k^{\prime}\right) .
\end{aligned}
$$

As $w\left(\mathbf{k}, \mathbf{k}^{\prime}\right)$ decays with $k^{-6}$ at larger values of $\mathbf{k}$, we can approximate the summation with an integral in $R\left(\mathbf{k}, k_{\text {int }}\right)$ :

$$
\begin{aligned}
R\left(\mathbf{k}, k_{\text {int }}\right) \approx & R_{I}\left(\mathbf{k}, k_{\text {int }}\right), \\
R_{I}\left(\mathbf{k}, k_{\text {int }}\right)= & \int_{k_{\text {int }}}^{\infty} k^{\prime 2} d k^{\prime} \int_{-1}^{1} d(\cos \theta) \frac{\left(k k^{\prime} \cos \theta-k^{\prime 2}\right)}{4 \pi^{2}} \\
& \times \tilde{u}\left(\sqrt{k^{2}-2 k k^{\prime} \cos \theta+k^{\prime 2}}\right) \tilde{u}\left(k^{\prime}\right) .
\end{aligned}
$$

Due to the additional conditions in the sum (E1), further restrictions apply at the boundaries of the integral, when

$$
\sqrt{k^{2}-2 k k^{\prime} \cos \theta+k^{\prime 2}}<k_{c} \text {. }
$$

In order to avoid the complicated limits of the integration, we choose $k_{\text {int }}$ large enough such that (E7) never occurs. After some algebra, it can be shown that it is sufficient to choose $k_{\text {int }}$ such as to satisfy

$$
k_{\text {int }} \geqslant k+k_{c},
$$

which is easy enough to fulfill as $k$ and $k_{c}$ are kept small to limit the size of the Hilbert space and to enhance the effect of the correlation factor.

In order to evaluate the integral (E6), we consider the Taylor-expanded form of $\tilde{u}(k)$ in Eq. (A1):

$$
\begin{aligned}
R_{I}\left(\mathbf{k}, k_{\text {int }}\right)= & -\frac{2 \pi^{2}}{k_{\text {int }}}-\frac{8 \pi}{a_{s} k_{\text {int }}^{2}}-\frac{32}{3 a_{s}^{2} k_{\text {int }}^{3}} \\
& -\frac{4 k^{2} \pi}{3 a_{s} k_{\text {int }}^{4}}+\mathcal{O}\left(a_{s}^{-2} k_{\text {int }}^{-5}\right) .
\end{aligned}
$$

In this paper we specifically consider unitary interactions, where the integral (E6) can be evaluated exactly:

$$
\lim _{a_{s} \rightarrow \pm \infty} R\left(\mathbf{k}, k_{\text {int }}\right) \approx \lim _{a_{s} \rightarrow \pm \infty} R_{I}\left(\mathbf{k}, k_{\text {int }}\right)=-\frac{2 \pi^{2}}{k_{\text {int }}} .
$$

In this work we have applied Eqs. (E2)-(E5) and (E9) to evaluate the matrix element $W(\mathbf{k})$ for up to $k=16 \pi / L$ with $k_{\text {int }}=1600 \pi / L$, where the finite sum $P\left(\mathbf{k}, k_{\text {int }}\right)$ was evaluated exactly and the infinite sum $R\left(\mathbf{k}, k_{\text {int }}\right)$ was replaced by the integral $R_{I}\left(\mathbf{k}, k_{\text {int }}\right)$. Varying $k$ up to the maximal value of $16 \pi / L$ we found the uncertainties in the values of $W(\mathbf{k})$ only in the seventh and eighth significant digits. As the energy scales linearly with the error in the matrix elements, the error should appear in the energy in the same order. Moreover, the accuracy of the integral approximation was also checked numerically by comparing the energies from $k_{\text {int }}=1200 \pi / L$ and $1600 \pi / L$ calculations. We did not find any significant difference in examples of two, three, and four fermions.

For two particles the convergence of the energy upon increasing $k_{\text {int }}$ is demonstrated in Fig. 6. The observed error seems adequate for our numerical calculations, where the uncertainty of our final results was in the fourth and fifth significant digits.

\section{APPENDIX F: DETAILS OF THE NUMERICAL CALCULATIONS}

\section{FCIQMC}

For the numerical calculation we used the NECI code [67], where transcorrelated Hamiltonians including three- 


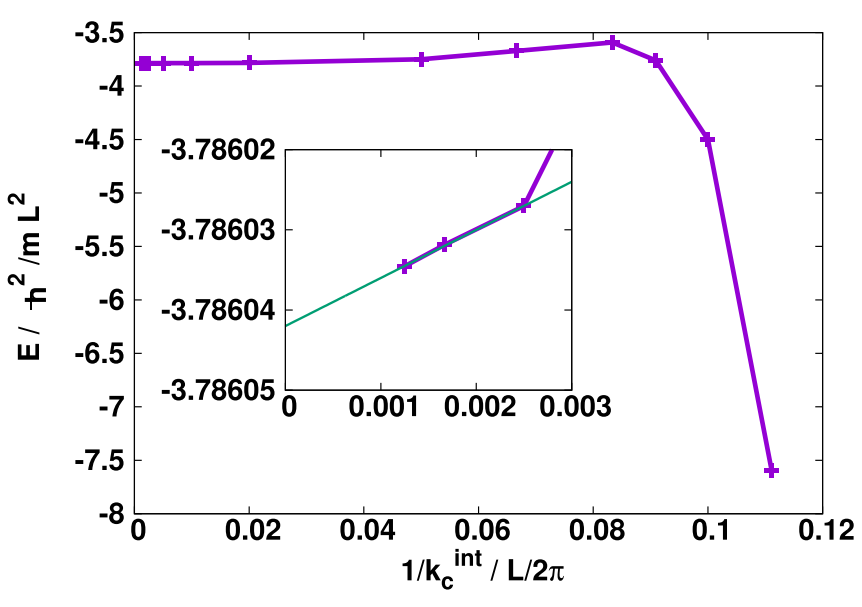

FIG. 6. Convergence of the energy of one spin-up and one spindown particle at unitary interaction with $k_{\text {int }}$. The maximal value of the momentum for the single-particle basis was $16 \pi / L$. The transcorrelated cutoff is kept to $k_{c}=2 \pi / L$. The inset shows a detail at enlarged scale. The extrapolation to $1 / k_{\text {int }}=0$ is determined with a linear fit (shown as straight line) to the last three data points.

body excitations had previously been implemented for the homogeneous electron gas [41], the Fermi-Hubbard model [52], atoms, molecules [42], and the Fermi gas in one dimension [43]. In the context of this project we have further extended the capabilities of the NECI code by including the transcorrelated Hamiltonian for the unitary Fermi gas in three dimensions.

For two particles, non-Hermitian exact (deterministic) diagonalization is applied in NECI using an external LAPACK library [68]. For three and four fermions the Hilbert space is too large for deterministic diagonalization. Hence, the full configuration interaction quantum Monte Carlo (FCIQMC) algorithm $[45,56]$ is applied to obtain the ground-state energy.

One of the elementary parameters of the FCIQMC algorithm is the number of the walkers [45,56]. It controls the resolution of the wave function and the memory usage of the algorithm. In this algorithm a minimal number of walkers is required to eliminate the sign problem.

The minimal number is determined by the annihilation plateau $[45,69]$, which appears in the number of walkers during the imaginary-time evolution. This plateau can be seen to appear in Fig. 7 at around 50000 walkers. At the end of the plateau, around $\tau \approx 5 \times 10^{6} E_{0}^{-1}$, the sign structure of the wave function is determined, fluctuations in the projected energy $E_{p}$ are greatly reduced, and the walker number starts growing exponentially. When the number of the walkers exceeds the initially set target walker number of $10^{6}$, we start adjusting the initially constant shift parameter $S$ according to protocol of Ref. [45] in order to control the walker number, which will subsequently fluctuate around a mean. Both the shift parameter $S$ as well as the projected energy $E_{p}$ provide estimators for the ground-state energy. The final value of the ground-state energy is determined by the mean of shift parameter (after reaching the final walker number). The error is obtained from an estimate of standard deviation of the mean using a standard blocking analysis to remove autocorrelations in the time series [70].
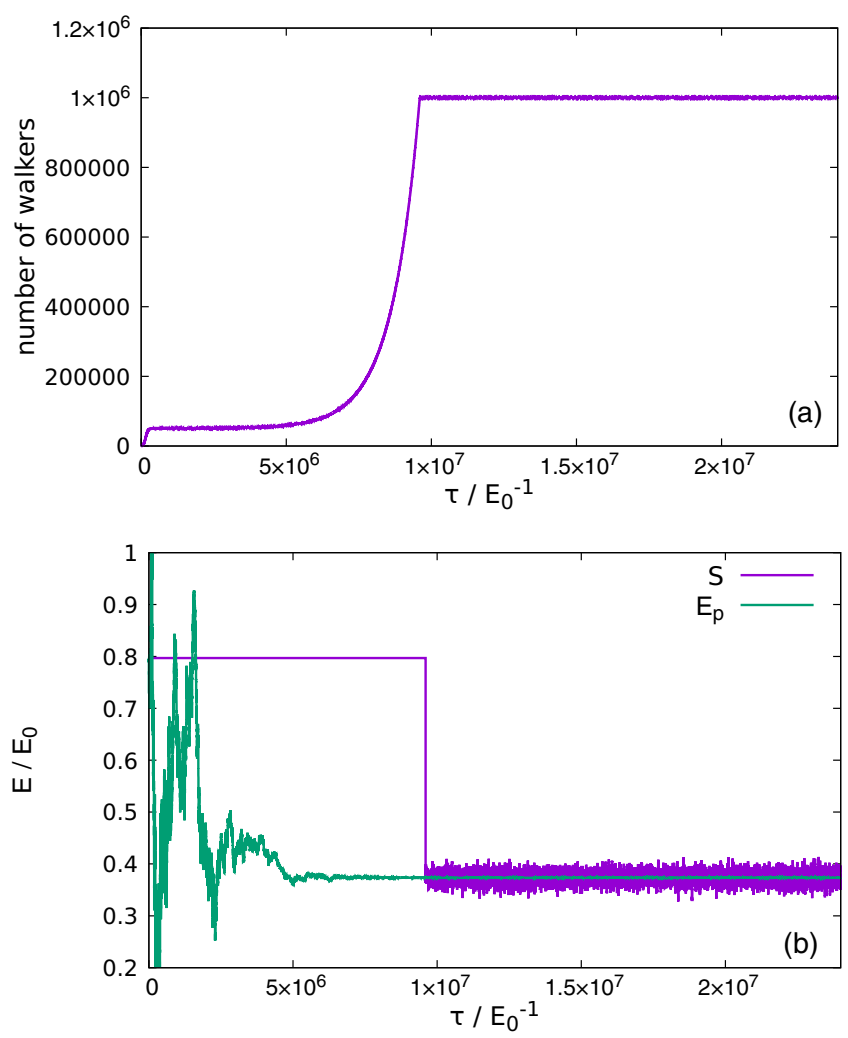

FIG. 7. (a) The total number of walkers and (b) the shift $S$ and projected energy $E_{p}$ during the FCIQMC simulation for the example of two spin-up and one spin-down particles with unitary interactions at $M=11^{3}$ using the transcorrelated approach at $k_{c}=2 \pi / L$. After the target walker number of $10^{6}$ is reached, the previously constant shift parameter is updated in order to control the walker number. $E_{0}=4 \pi^{2} \hbar^{2} / m L^{2}$ is the noninteracting energy.

For all calculations for three fermions and for the latticerenormalized calculations for four fermions, we were able to apply a large enough walker number to detect and exceed the annihilation plateau. However, for the transcorrelated calculations with four fermions with $M>9^{3}$ the annihilation plateau was too high for the available numerical resources. In these cases we applied the initiator method [61], which has proved to be efficient for electronic structure calculations $[62,71,72]$. While this approximation causes a systematic bias in the calculations, the bias disappears when increasing the number of walkers. For all results shown, the number of walkers was increased until the changes in energy were insignificant compared to the statistical error bars. Another systematic bias, the population control bias, is known to affect FCIQMC calculations with small walker number but is well below the statistical error for the parameters considered in our calculations. We thus expect the FCIQMC results presented in this work to be essentially free of any systematic bias.

The parameters for the calculations are shown in Tables I and II. The calculations were typically run on a single node with 20 or 40 processor cores for 3 to 9 days. The largest calculation was for four particles with $k_{c}=4 \pi / L$ and $M=17^{3}$. The memory usage in this example was about 114 GByte and about 351 days of CPU time were used. 
TABLE I. Parameters of the FCIQMC calculation for two spinup and one spin-down particles. $N_{w}$ is the number of walkers and $N_{\tau}$ is the number of time steps. The time-step size $\Delta \tau$ was determined by the histogram $\tau$ search algorithm [67]. An annihilation plateau was detected for all three-particle calculations.

\begin{tabular}{lcrrl}
\hline \hline$k_{c} / 2 \pi L^{-1}$ & $M$ & \multicolumn{1}{c}{$N_{w}$} & \multicolumn{1}{c}{$N_{\tau}$} & \multicolumn{1}{c}{$\Delta \tau / E_{0}^{-1}$} \\
\hline 1 & $11^{3}$ & 10000000 & 1310720 & 0.00010 \\
1 & $13^{3}$ & 16000000 & 327680 & 0.000067 \\
1 & $15^{3}$ & 20000000 & 327680 & 0.000039 \\
2 & $13^{3}$ & 2000000 & 1310720 & 0.00039 \\
2 & $15^{3}$ & 10000000 & 163840 & 0.00018 \\
2 & $17^{3}$ & 20000000 & 327680 & 0.00011 \\
\hline \hline
\end{tabular}

\section{Complete basis limit and uncertainty}

The complete basis limit of the energy $E_{\mathrm{cb}}$ can be determined by extrapolation assuming that the asymptotic scaling of the energy $E$ with the size of the basis set is known. According to the observed scaling of the two-particle energy seen in Fig. 2 and discussed in Sec. III, we expect the basis-set error of the energy to be inversely proportional to the number $M$ of plane-wave modes. Thus,

$$
E / E_{0}=\alpha+\beta \frac{1}{M},
$$

where $\alpha=E_{\mathrm{cb}} / E_{0}$ and $\beta$ are dimensionless fitting parameters. Linear fits to Eq. (F1) are shown in Fig. 4 for three-fermion data and in Fig. 8 for four-fermion data, where $\alpha$ and $\beta$ represent the slope and intersect of the fitted lines, respectively. Estimators for the mean values of $\alpha$ and $\beta$, their variances $\sigma_{\alpha}^{2}$ and $\sigma_{\beta}^{2}$, and their covariance $\operatorname{cov}(\alpha, \beta)$ are obtained using $\chi^{2}$ fitting [73]. This assumes that each data point is a Gaussian random variable with standard deviation given by the error bar, as justified in Monte Carlo simulations.

Since we assume the linear relationship (F1) to hold only asymptotically for large $M$, we have to decide which data points to include in the linear fit. We expect our calculations with the smaller cutoff parameter $k_{c}=2 \pi / L$ to enter the asymptotic regime for smaller $M$ compared to the larger value $k_{c}=4 \pi / L$ because of the larger correlation factor. We also expect simulations with both values of $k_{c}$ to share the same limit $E_{\mathrm{cb}}$, and thus independently extrapolate to the same intersect. Thus, we first consider the data for the smaller cutoff value $k_{c}=2 \pi / L$ and choose the smallest value of $M$ above

TABLE II. Parameters of the FCIQMC calculation for two spinup and two spin-down particles, as in Table I. An annihilation plateau was detected for the calculation with $M=9^{3}$. All other calculations were performed using the initiator approach [61].

\begin{tabular}{lcrrl}
\hline \hline$k_{c} / 2 \pi L^{-1}$ & $M$ & \multicolumn{1}{c}{$N_{w}$} & \multicolumn{1}{c}{$N_{\tau}$} & \multicolumn{1}{c}{$\Delta \tau / E_{0}^{-1}$} \\
\hline 1 & $9^{3}$ & 2000000 & 655360 & 0.000079 \\
1 & $11^{3}$ & 8000000 & 1310720 & 0.000051 \\
1 & $13^{3}$ & 16000000 & 1310720 & 0.0000079 \\
2 & $13^{3}$ & 9500000 & 1310720 & 0.0000079 \\
2 & $15^{3}$ & 16000000 & 327680 & 0.000051 \\
2 & $17^{3}$ & 160000000 & 327680 & 0.000029 \\
\hline \hline
\end{tabular}

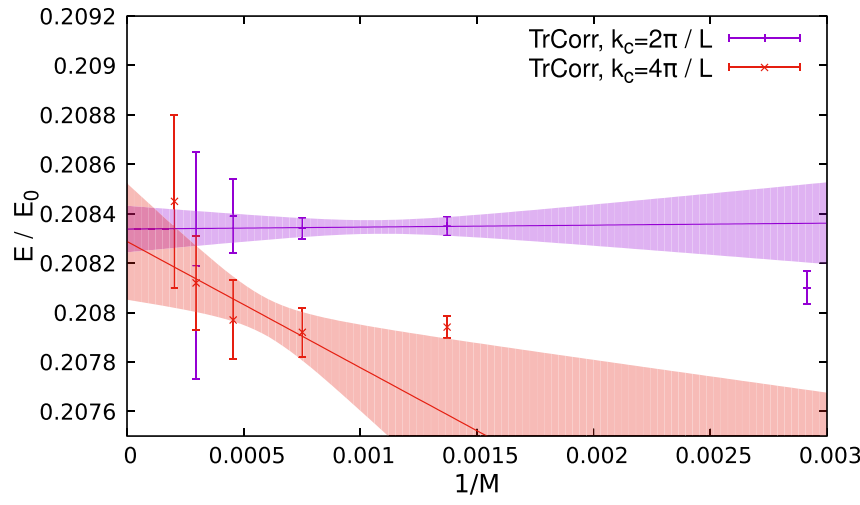

FIG. 8. The ground-state energy of two spin-up and two spindown fermions. The purple (upper) and red (lower) line and band show the linear fit with $1 \sigma$ confidence band obtained from $\chi^{2}$ fitting for $k_{c}=2 \pi / L\left(M=9^{3}, 11^{3}, 13^{3}, 15^{3}\right)$, and for $k_{c}=4 \pi / L(M=$ $\left.11^{3}, 13^{3}, 15^{3}, 17^{3}\right)$, respectively. $E_{0}=4 \pi^{2} \hbar^{2} / m L^{2}$ is the lowest noninteracting energy in the zero momentum sector.

which all data points for the energy $E$ reasonably represent the linear relationship (F1). For Fig. 4 this corresponds to $M=9^{3}(1 / M \approx 0.0014)$ and yields four data points. Then, we consider the data for the larger value $k_{c}=4 \pi / L$ and choose the largest $M$ such that the intersects for interpolation with both cutoff values are consistent within the one- $\sigma$ confidence interval. For Fig. 4 this yields three data points at $k_{c}=4 \pi / L$ with $M \geqslant 11^{3}$. For the four-fermion data in Fig. 8 the same procedure yields four data points for $k_{c}=2 \pi / L$ and four data points for $k_{c}=4 \pi / L$. It can be seen from the figures that the procedure is successful and the intercepts have overlapping confidence intervals. For both three- and four-fermion data sets, the extrapolations with the smaller cutoff value $k_{c}=$ $2 \pi / L$ yield the smaller confidence intervals for the complete basis-set limit $E_{\mathrm{cb}}$, and thus the corresponding values are reported as the final results.

The results of the complete basis-set extrapolation for fourfermion calculations are compared with literature results in Fig. 5 and in Table III. The error bands shown in Figs. 4 and 8 were calculated as the $1 \sigma$ confidence intervals for the energy for each value of $1 / M$ using the following procedure. The $1 \sigma$ confidence interval at the given value of $x=1 / M$ can be

TABLE III. Numerical values of the data shown in Fig. 5. Ground-state energies for two spin-up and two spin-down particles. The renormalization methodology for the different dispersion relations is described in Appendix F 4 and follows Refs. [18,19].

\begin{tabular}{lll}
\hline \hline Method & $E / E_{0}$ & $\mathrm{SE}\left(E / E_{0}\right)$ \\
\hline Transcorrelated FCIQMC & 0.208338 & 0.000094 \\
Hubbard FCIQMC & 0.2087 & 0.0021 \\
Quadratic dispersion FCIQMC & 0.2087 & 0.0011 \\
Endres 1 AFQMC $\mathcal{O}(4)$ [20] & 0.2122 & 0.0040 \\
Endres 2 AFQMC O(5) [20] & 0.2130 & 0.0026 \\
Bour 1 AFQMC [59] & 0.211 & 0.002 \\
Bour 2 AFQMC 2 [59] & 0.210 & 0.002 \\
Bour 3 AFQMC Euclidian [59] & 0.206 & 0.009 \\
Yin ECG [33] & 0.2058 & 0.0021 \\
\hline \hline
\end{tabular}




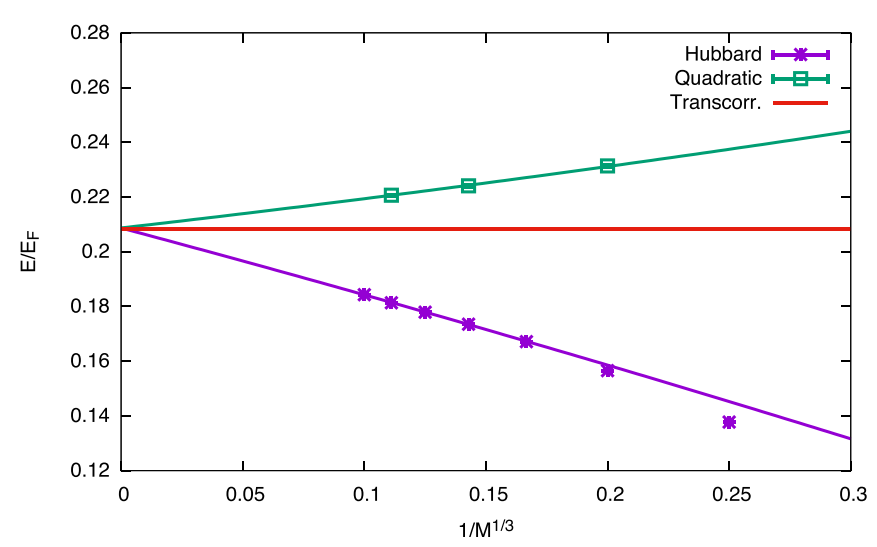

FIG. 9. Fitting procedure for results obtained using the renormalized lattice method with different single-particle dispersions. The value obtained using the transcorrelated method is included for comparison as the horizontal red line, with the error being smaller than the linewidth in this plot. The quadratic dispersion leads to two sets of points, depending on whether $M$ is even or odd. Here, we only show the results for odd values, which are considerably closer to the final extrapolated result.

calculated from the standard deviation $\sigma_{a}(x)$ of the intercept $a$ in the linear equation

$$
E / E_{0}=a+b\left(\frac{1}{M}-x\right) .
$$

The $68 \%$ or $1 \sigma$ confidence interval is then given as

$$
a-\sigma_{a}(x)<\frac{E}{E_{0}}<a+\sigma_{a}(x) .
$$

The new parameters $a$ and $b$ can be expressed by the original parameters in Eq. (F1):

$$
\begin{gathered}
a=\alpha+\beta x, \\
b=\beta .
\end{gathered}
$$

Using Eq. (F4), the standard deviation of parameter $a$ is then determined as [73]

$$
\sigma_{a}(x)=\sqrt{\sigma_{\alpha}^{2}+2 \operatorname{cov}(\alpha, \beta) x+\sigma_{\beta}^{2} x^{2}} .
$$

\section{Extrapolated values for four particles in renormalized lattice calculations}

In the main text we present extrapolated values for the ground-state energy for four fermions obtained using the renormalized lattice method with the standard Hubbard dispersion and a quadratic dispersion in Fig. 5. These dispersions lead to a dominant convergence rate proportional to $M^{-1 / 3}$, as can be seen in Fig. 9. We fit a function $f(M)=\frac{E}{E_{0}}+$ $A M^{-1 / 3}+B M^{-2 / 3}$ into the FCIQMC results to obtain an extrapolated value for $E / E_{0}$ with fitting error.

\section{Dispersions for renormalized lattice calculations}

A useful basis-set expansion for a free-space Fermi system is using the Hubbard model in the low-density limit. In this regime, any single-particle dispersion which is quadratic
TABLE IV. Single-particle dispersions as functions of lattice momentum $\vec{k}$ used in FCIQMC simulations in this work for comparison with the transcorrelated method. Here, $U$ and $t$ denote the usual Hubbard interaction and hopping parameters, $\alpha$ is the lattice constant. The numerical constants are $C_{1}=0.257022, C_{2}=$ $-12.89076, C_{3}=-1.728219$, and $X=\frac{1}{6}\left[\sum_{i=1}^{3} 1-\cos \left(k_{\alpha}\right)\right]$. All functions and numerical values can be found with more details in Ref. [18].

\begin{tabular}{lcr}
\hline \hline Name & Function & \multicolumn{1}{c}{$U / t$} \\
\hline Hubbard & $2 t \sum_{i=1}^{3}\left[1-\cos \left(k_{i} \alpha\right)\right]$ & -7.91355 \\
Quadratic & $t(\vec{k} \alpha)^{2}$ & -10.28871 \\
Quartic & $t(\vec{k} \alpha)^{2}\left[1-C_{1}\left(\frac{\vec{k} \alpha}{\pi}\right)^{2}\right]$ & -8.66661 \\
Magic & $12 t X\left(1+C_{2} X+C_{3} X^{2}\right)$ & -12.89076 \\
\hline \hline
\end{tabular}

around zero will converge to the same result in the infinitebasis states (lattice sites) limit [57]. Werner and Castin have proposed to replace the standard Hubbard single-particle dispersion with several candidates which eliminate finite effective-range contributions to varying degrees [18]. This leads to a convergence rate improvement from $M^{-1 / 3}$ for the Hubbard and quadratic dispersions to $M^{-2 / 3}$ for so-called "magic" dispersions. While a quartic dispersion was fitted to converge with the same power law as the magic dispersion in Ref. [19], for the smaller particle numbers in our case we find a small contribution remaining that is proportional to $M^{-1 / 3}$. In Table IV, we list all dispersions used in this work with the renormalized Hubbard interaction parameters corresponding to unitary interactions.

\section{Effective three-body interaction terms}

Evaluating the transcorrelated Hamiltonian during any diagonalization procedure requires increased numerical effort compared to the renormalized lattice Hamiltonian. The largest part of the increased effort can be attributed to the three-body term and thus scales with $N^{3}$, where $N$ is the number of particles. An efficient procedure for implementing the three-body term in the FCIQMC algorithm is described in Ref. [52]. By sampling the three-body interactions at a lower rate than the much stronger and more important two-body interactions, the increased computational effort for having more nonzero offdiagonal matrix elements can be mostly mitigated, leaving a modest additional cost for treating the three-body-interaction terms explicitly.

The results presented in the main part of the paper were computed by fully including all three-body excitations. In the following we discuss an approximate procedure previously used in Refs. [41,43] that only requires evaluating effective two-body matrix elements, and thus reduces the numerical effort further, while still producing highly accurate results. Specifically, for our three-particle calculations the computation time reduced by a factor of 2-3 with approximated three-body terms (effective two-body interactions only) compared to explicitly evaluating all three-body terms. For the four-particle calculation, the speedup factor was approximately 3-4. 


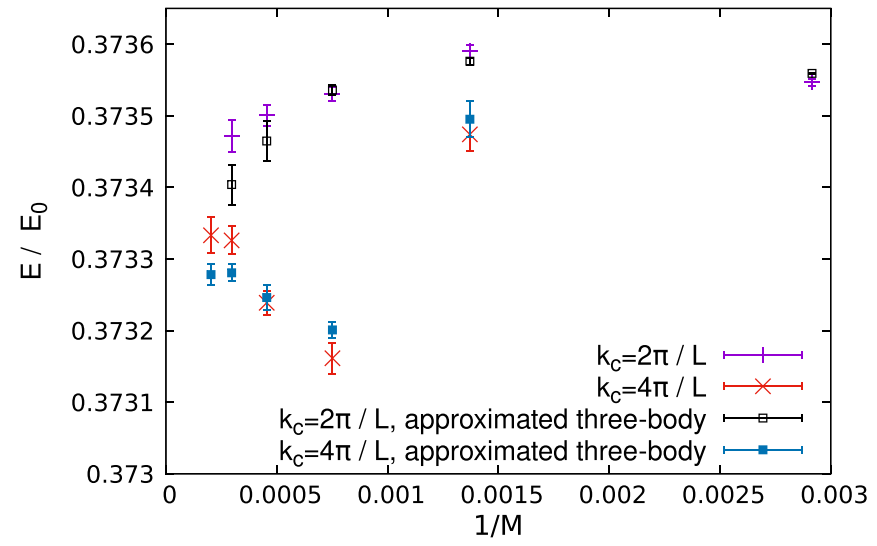

FIG. 10. The lowest energy of two spin-up and one spin-down fermions in the zero-momentum sector with the full transcorrelated Hamiltonian and with the approximated three-body term as per Eq. (F7). $E_{0}=4 \pi^{2} \hbar^{2} / m L^{2}$ is the energy with zero interaction between the fermions.

The approximate three-body interactions work by only allowing excitations that change not more than two singleparticle orbitals in the Fock state $|\Phi\rangle$ :

$$
\begin{aligned}
& \sum_{\substack{\mathbf{p q s} \\
\mathbf{k} \mathbf{k}^{\prime} \\
\sigma}} Q_{\mathbf{k} \mathbf{k}^{\prime}} a_{\mathbf{p}-\mathbf{k}, \sigma}^{\dagger} a_{\mathbf{q}+\mathbf{k}^{\prime}, \sigma}^{\dagger} a_{\mathbf{s}+\mathbf{k}-\mathbf{k}^{\prime}, \bar{\sigma}}^{\dagger} a_{\mathbf{s}, \bar{\sigma}} a_{\mathbf{q}, \sigma} a_{\mathbf{p}, \sigma}|\Phi\rangle \\
& \approx \sum_{\mathbf{p q}} N_{\bar{\sigma}} Q_{\mathbf{k k}} a_{\mathbf{p}-\mathbf{k}, \sigma}^{\dagger} a_{\mathbf{q}+\mathbf{k}, \sigma}^{\dagger} a_{\mathbf{q}, \sigma} a_{\mathbf{p}, \sigma}|\Phi\rangle \\
& \quad-\sum_{\mathbf{k} \sigma} N_{\sigma} Q_{\mathbf{p}-\mathbf{q}, \mathbf{k}} a_{\mathbf{p}-\mathbf{k}, \sigma}^{\dagger}\left(a_{\mathbf{s}+\mathbf{p}-\mathbf{q}+\mathbf{k}, \bar{\sigma}}^{\dagger}+a_{\mathbf{s}+\mathbf{q}-\mathbf{p}+\mathbf{k}, \bar{\sigma}}^{\dagger}\right) \\
& \quad \times a_{\mathbf{k}, \bar{\sigma}}^{\dagger} a_{\mathbf{p}, \sigma}|\Phi\rangle,
\end{aligned}
$$

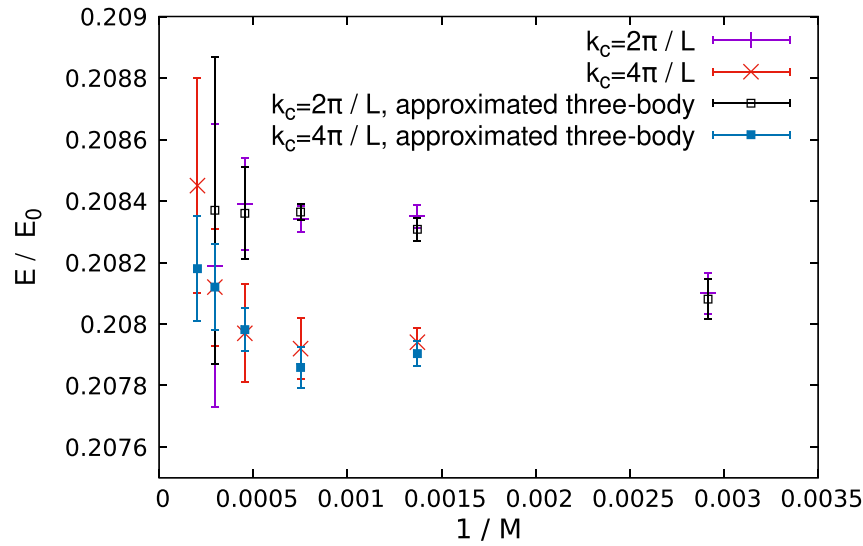

FIG. 11. The ground-state energy of two spin-up and two spindown fermions with the full transcorrelated Hamiltonian and with the approximated three-body term as per Eq. (F7). $E_{0}=4 \pi^{2} \hbar^{2} / m L^{2}$ is the noninteracting energy.

where $\sigma \neq \bar{\sigma}, N_{\sigma}$ is the number of the particles with spin $\sigma$, and we used the identity $\sum_{r} a_{r, \sigma}^{\dagger} a_{r, \sigma}|\Phi\rangle=N_{\sigma}|\Phi\rangle$. This approximation is closely related to the random phase approximation (RPA) [74-76].

Ground-state energies computed with approximated threebody terms are compared to the full transcorrelated Hamiltonian in Fig. 10 for three fermions and in Fig. 11 for four fermions. We find that the approximate results and the full transcorrelated results have mostly overlapping Monte Carlo (statistical) error bars. The difference between full and approximated three-body terms is not statistically significant. We thus conclude that the error made by approximating the three-body terms with the RPA-like right-hand side of Eq. (F7) is below the statistical Monte Carlo error for our calculations.
[1] The BCS-BEC Crossover and the Unitary Fermi Gas, Lecture Notes in Physics, edited by W. Zwerger (Springer, Berlin, 2012), Vol. 836.

[2] M. J. H. Ku, A. T. Sommer, L. W. Cheuk, and M. W. Zwierlein, Revealing the superfluid lambda transition in the universal thermodynamics of a unitary fermi gas, Science 335, 563 (2012).

[3] B. Mukherjee, Z. Yan, P. B. Patel, Z. Hadzibabic, T. Yefsah, J. Struck, and M. W. Zwierlein, Homogeneous Atomic Fermi Gases, Phys. Rev. Lett. 118, 123401 (2017).

[4] C. Eigen, J. A. P. Glidden, R. Lopes, E. A. Cornell, R. P. Smith, and Z. Hadzibabic, Universal prethermal dynamics of Bose gases quenched to unitarity, Nature (London) 563, 221 (2018).

[5] C. Carcy, S. Hoinka, M. G. Lingham, P. Dyke, C. C. N. Kuhn, $\mathrm{H}$. $\mathrm{Hu}$, and C. J. Vale, Contact And Sum Rules In A NearUniform Fermi Gas At Unitarity, Phys. Rev. Lett. 122, 203401 (2019).

[6] B. Mukherjee, P. B. Patel, Z. Yan, R. J. Fletcher, J. Struck, and M. W. Zwierlein, Spectral Response And Contact Of The Unitary Fermi Gas, Phys. Rev. Lett. 122, 203402 (2019).
[7] A. N. Wenz, G. Zürn, S. Murmann, I. Brouzos, T. Lompe, and S. Jochim, From few to many: Observing the formation of a fermi sea one atom at a time, Science 342, 457 (2013).

[8] L. A. Reynolds, E. Schwartz, U. Ebling, M. Weyland, J. Brand, and M. F. Andersen, Direct Measurements Of Collisional Dynamics In Cold Atom Triads, Phys. Rev. Lett. 124, 073401 (2020).

[9] J. Weiner, V. S. Bagnato, S. Zilio, and P. S. Julienne, Experiments and theory in cold and ultracold collisions, Rev. Mod. Phys. 71, 1 (1999).

[10] E. Braaten and H.-W. Hammer, Universality in few-body systems with large scattering length, Phys. Rep. 428, 259 (2006).

[11] S. Giorgini, L. P. Pitaevskii, and S. Stringari, Theory of ultracold atomic fermi gases, Rev. Mod. Phys. 80, 1215 (2008).

[12] H. Bethe and R. Peierls, Quantum theory of the diplon, Proc. R. Soc. London, Ser. A 148, 146 (1935). 
[13] T. Busch, B.-G. Englert, K. Rzażewski, and M. Wilkens, Two cold atoms in a harmonic trap, Found. Phys. 28, 549 (1998).

[14] D. S. Petrov, C. Salomon, and G. V. Shlyapnikov, Weakly Bound Dimers Of Fermionic Atoms, Phys. Rev. Lett. 93, 090404 (2004).

[15] F. Werner and Y. Castin, Unitary Quantum Three-Body Problem In A Harmonic Trap, Phys. Rev. Lett. 97, 150401 (2006).

[16] X.-Ji. Liu, H. Hu, and P. D. Drummond, Three attractively interacting fermions in a harmonic trap: Exact solution, ferromagnetism, and high-temperature thermodynamics, Phys. Rev. A 82, 023619 (2010).

[17] A. Deltuva, Universality in fermionic dimer-dimer scattering, Phys. Rev. A 96, 022701 (2017).

[18] F. Werner and Y. Castin, General relations for quantum gases in two and three dimensions: Two-component fermions, Phys. Rev. A 86, 013626 (2012).

[19] J. Carlson, S. Gandolfi, K. E. Schmidt, and S. Zhang, Auxiliary-field quantum Monte Carlo method for strongly paired fermions, Phys. Rev. A 84, 061602(R) (2011).

[20] M. G. Endres, D. B. Kaplan, J.-W. Lee, and A. N. Nicholson, Lattice Monte Carlo calculations for unitary fermions in a finite box, Phys. Rev. A 87, 023615 (2013).

[21] D. Lee, Ground state energy at unitarity, Phys. Rev. C 78, 024001 (2008).

[22] R. He, N. Li, B.-N. Lu, and D. Lee, Superfluid condensate fraction and pairing wave function of the unitary fermi Gas, Phys. Rev. A 101, 063615 (2020).

[23] D. Lee and T. Schäfer, Cold dilute neutron matter on the lattice. II. Results in the unitary limit, Phys. Rev. C 73, 015202 (2006).

[24] E. Burovski, N. Prokofeev, B. Svistunov, and M. Troyer, The fermi-hubbard model at unitarity, New J. Phys. 8, 153 (2006).

[25] A. Bulgac, J. E. Drut, and P. Magierski, Quantum Monte Carlo simulations of the BCS-BEC crossover at finite temperature, Phys. Rev. A 78, 023625 (2008).

[26] O. Goulko and M. Wingate, Thermodynamics of balanced and slightly spin-imbalanced Fermi gases at unitarity, Phys. Rev. A 82, 053621 (2010).

[27] O. Goulko and M. Wingate, Numerical study of the unitary Fermi gas across the superfluid transition, Phys. Rev. A 93, 053604 (2016).

[28] L. Rammelmüller, A. C. Loheac, J. E. Drut, and J. Braun, Finite-Temperature Equation of State of Polarized Fermions at Unitarity, Phys. Rev. Lett. 121, 173001 (2018).

[29] S. Jensen, C. N. Gilbreth, and Y. Alhassid, The Contact in the Unitary Fermi Gas Across the Superfluid Phase Transition, Phys. Rev. Lett. 125, 043402 (2020).

[30] S. Jensen, C. N. Gilbreth, and Y. Alhassid, Pairing Correlations Across The Superfluid Phase Transition In The Unitary Fermi Gas, Phys. Rev. Lett. 124, 090604 (2020).

[31] A. Richie-Halford, J. E. Drut, and A. Bulgac, Emergence of a pseudogap in the BCS-BEC crossover, Phys. Rev. Lett. 125, 060403 (2020).

[32] I. Stetcu, B. R. Barrett, U. van Kolck, and J. P. Vary, Effective theory for trapped few-fermion systems, Phys. Rev. A 76, 063613 (2007).

[33] X. Y. Yin and D. Blume, Small two-component Fermi gases in a cubic box with periodic boundary conditions, Phys. Rev. A 87, 063609 (2013).

[34] P. Jeszenszki, A. Yu. Cherny, and J. Brand, s-wave scattering length of a Gaussian potential, Phys. Rev. A 97, 042708 (2018).
[35] P. Jeszenszki, A. Alavi, and J. Brand, Are smooth pseudopotentials a good choice for representing short-range interactions? Phys. Rev. A 99, 033608 (2019).

[36] Y. Alhassid, G. F. Bertsch, and L. Fang, New Effective Interaction For The Trapped Fermi Gas, Phys. Rev. Lett. 100, 230401 (2008).

[37] J. Christensson, C. Forssén, S. Åberg, and S. M. Reimann, Effective-interaction approach to the many-boson problem, Phys. Rev. A 79, 012707 (2009).

[38] S. F. Boys and N. C. Handy, The determination of energies and wavefunctions with full electronic correlation, Proc. R. Soc. A 310, 43 (1969).

[39] O. Hino, Y. Tanimura, and S. Ten-no, Application of the transcorrelated Hamiltonian to the linearized coupled cluster singles and doubles model, Chem. Phys. Lett. 353, 317 (2002).

[40] S. Ten-no and O. Hino, New transcorrelated method improving the feasibility of explicitly correlated calculations, Int. J. Mol. Sci. 3, 459 (2002).

[41] H. Luo and A. Alavi, Combining the transcorrelated method with full configuration interaction quantum monte carlo: Application to the homogeneous electron gas, J. Chem. Theory Comput. 14, 1403 (2018).

[42] A. J. Cohen, H. Luo, K. Guther, W. Dobrautz, D. P. Tew, and A. Alavi, Similarity transformation of the electronic Schrödinger equation via Jastrow factorisation, J. Chem. Phys. 151, 061101 (2019).

[43] P. Jeszenszki, H. Luo, A. Alavi, and J. Brand, Accelerating the convergence of exact diagonalization with the transcorrelated method: Quantum gas in one dimension with contact interactions, Phys. Rev. A 98, 053627 (2018).

[44] T. Kato, On the eigenfunctions of many-particle systems in quantum mechanics, Commun. Pure Appl. Math. 10, 151 (1957).

[45] G. H. Booth, A. J. W. Thom, and A. Alavi, Fermion Monte Carlo without fixed nodes: A game of life, death, and annihilation in Slater determinant space, J. Chem. Phys. 131, 054106 (2009).

[46] K. Huang and C. N. Yang, Quantum-mechanical many-body problem with hard-sphere interaction, Phys. Rev. 105, 767 (1957).

[47] W. Yi, Y. Han, and W. Zhang, Physics on Ultracold Quantum Gases, Peking University-World Scientific Advanced Physics Series (World Scientific, New Jersey, 2018).

[48] M. Olshanii and L. Pricoupenko, Rigorous Approach to the Problem of Ultraviolet Divergencies in Dilute Bose Gases, Phys. Rev. Lett. 88, 010402 (2001).

[49] B. D. Esry and C. H. Greene, Validity of the shape-independent approximation for Bose-Einstein condensates, Phys. Rev. A 60 , 1451 (1999).

[50] M. Rontani, G. Eriksson, S. Åberg, and S. M. Reimann, On the renormalization of contact interactions for the configurationinteraction method in two-dimensions, J. Phys. B: At., Mol. Opt. Phys. 50, 065301 (2017).

[51] R. A. Doganov, S. Klaiman, O. E. Alon, A. I. Streltsov, and L. S. Cederbaum, Two trapped particles interacting by a finiterange two-body potential in two spatial dimensions, Phys. Rev. A 87, 033631 (2013).

[52] W. Dobrautz, H. Luo, and A. Alavi, Compact numerical solutions to the two-dimensional repulsive Hubbard model obtained 
via nonunitary similarity transformations, Phys. Rev. B 99, 075119 (2019).

[53] A. Köhn and A. Tajti, Can coupled-cluster theory treat conical intersections? J. Chem. Phys. 127, 044105 (2007).

[54] V. Efimov, Energy levels arising from resonant two-body forces in a three-body system, Phys. Lett. B 33, 563 (1970).

[55] P. Naidon and S. Endo, Efimov physics: A review, Rep. Prog. Phys. 80, 056001 (2017).

[56] G. H. Booth, S. D. Smart, and A. Alavi, Linear-scaling and parallelisable algorithms for stochastic quantum chemistry, Mol. Phys. 112, 1855 (2014).

[57] L. Pricoupenko and Y. Castin, Three fermions in a box at the unitary limit: Universality in a lattice model, J. Phys. A: Math. Theor. 40, 12863 (2007).

[58] Y. Castin, Basic theory tools for degenerate Fermi gases, in Utracold Fermi Gases, Proceedings of Enrico Fermi Varenna School of Physics, edited by M. Inguscio, W. Ketterle, and C. Salomon (IOS Press, Amsterdam, 2007), p. 60.

[59] S. Bour, X. Li, D. Lee, Ulf-G. Meißner, and L. Mitas, Precision benchmark calculations for four particles at unitarity, Phys. Rev. A 83, 063619 (2011).

[60] The accurate three-particle energy with standard error was received by private communication from Ludovic Pricoupenko and Yvan Castin based on Ref. [57].

[61] D. Cleland, G. H. Booth, and A. Alavi, Communications: Survival of the fittest: Accelerating convergence in full configuration-interaction quantum Monte Carlo, J. Chem. Phys. 132, 041103 (2010).

[62] G. H. Booth, A. Grüneis, G. Kresse, and A. Alavi, Towards an exact description of electronic wavefunctions in real solids, Nature (London) 493, 365 (2013).

[63] K. Ghanem, A. Y. Lozovoi, and A. Alavi, Unbiasing the initiator approximation in full configuration interaction quantum Monte Carlo, J. Chem. Phys. 151, 224108 (2019).

[64] N. S. Blunt, T. W. Rogers, J. S. Spencer, and W. M. C. Foulkes, Density-matrix quantum Monte Carlo method, Phys. Rev. B 89, 245124 (2014).
[65] K. Guther, W. Dobrautz, O. Gunnarsson, and A. Alavi, Time Propagation and Spectroscopy of Fermionic Systems Using a Stochastic Technique, Phys. Rev. Lett. 121, 056401 (2018).

[66] A. Nagy and V. Savona, Driven-dissipative quantum Monte Carlo method for open quantum systems, Phys. Rev. A 97, 052129 (2018).

[67] Neci, (2013), codebase on GitHub, https://github.com/ghb24/ NECI_STABLE.

[68] LAPACK Users' Guide, 3rd ed., edited by E. Anderson (Society for Industrial and Applied Mathematics, Philadelphia, 1999).

[69] J. S. Spencer, N. S. Blunt, and W. M. C. Foulkes, The sign problem and population dynamics in the full configuration interaction quantum Monte Carlo method, J. Chem. Phys. 136, 054110 (2012).

[70] H. Flyvbjerg and H. G. Petersen, Error estimates on averages of correlated data, J. Chem. Phys. 91, 461 (1989).

[71] J. J. Shepherd, G. H. Booth, and A. Alavi, Investigation of the full configuration interaction quantum Monte Carlo method using homogeneous electron gas models, J. Chem. Phys. 136, 244101 (2012).

[72] G. Li Manni, S. D. Smart, and A. Alavi, Combining the complete active space self-consistent field method and the full configuration interaction quantum monte carlo within a super-CI framework, with application to challenging metalPorphyrins, J. Chem. Theory Comput. 12, 1245 (2016).

[73] Numerical Recipes: The Art of Scientific Computing, 3rd ed., edited by W. H. Press (Cambridge University Press, Cambridge, UK, 2007).

[74] T. Gaskell, The collective treatment of a fermi gas: II, Proc. Phys. Soc. 77, 1182 (1961).

[75] E. A. G. Armour, The calculation of the ground-state energy of the free-electron gas by the transcorrelated method, J. Phys. C: Solid State Phys. 13, 343 (1980).

[76] A. L. Fetter and J. D. Walecka, Quantum Theory of Manyparticle Systems, Dover Books on Physics (Dover, New York, 2003), p. 601. 